Chapter 12

\title{
Surgical Treatment of Nevi and Melanoma in the Pediatric Age
}

\author{
Andrea Zangari, Federico Zangari, \\ Mercedes Romano, Elisabetta Cerigioni, \\ Maria Giovanna Grella, Anna Chiara Contini and \\ Martino Ascanio
}

Additional information is available at the end of the chapter

http://dx.doi.org/10.5772/54632

\section{Introduction}

Surgical care of children affected by melanocytic lesions is a complex area of pediatric surgery, where psychosocial aspects, involving parents and children of different ages, overlap with oncologic implications. In this challenging field results may be frustrating despite knowledge and experience.

Due to its rarity, the occurrence of malignant melanoma in children may be underestimated by involved professionals. Melanoma in children is rare, but it does exist and every effort should be done to assure proper treatment, which should be guaranteed in a pediatric tertiary care center by a multi-specialist approach.

The role of dermatologists is essential to achieve proper selection of indications to surgery, by clinical follow up and dermatoscopy techniques. On the other hand surgeon is expected to share a profound knowledge of indications and techniques of treatment. In fact, the awareness that simple excision of a nevus may represent the first and most important therapeutic intervention of a MM, before knowing the definitive diagnosis, is sometimes lacking even among pediatric care professionals and the occasional occurrence of a MM diagnosis may be confounding and cause of an incomplete care strategy.

Decisions about surgical treatment of congenital giant nevi in some cases may need psychological assessment because of complex relationships between patient's and parents' awareness and willingness. 
Pediatric anesthesia offers a variety of techniques that can be personalized to suite patients from the newborn to the adolescent.

The intent of this chapter is to contribute to knowledge of this multifaceted field of pediatric surgery.

\section{Nevomelanocytic lesions in the pediatric age}

Current strategies for the treatment of nevomelanocytic lesions in children mostly derived from the more extensive experience in adults. Nevertheless, further knowledge and experience in this field have shown some peculiarities that require special considerations relevant to the pediatric age. In fact, due to the multifaceted field of congenital nevi, to the rarity of melanoma in children and to peculiar features of some nevic lesions in this age range, important implications related to treatment emerge.

\subsection{Congenital nevi}

Congenital nevi are present at birth and occur approximately in $1 \%$ of newborn infants. They result from a proliferation of benign melanocytes in the dermis, epidermis, or both. Occasionally, nevi that are histologically identical to congenital nevi may develop approximately during the first 2 years of life. These are referred to be considered tardive congenital nevi [1].

The etiology of congenital melanocytic nevi remains unclear. The melanocytes of the skin originate in the neuroectoderm, although the specific cell type from which they derive remains controversial $[3,4,5]$.

One hypothesis is that pluripotential nerve sheath precursor cells migrate from the neural crest to the skin along paraspinal ganglia and peripheral nerve sheaths and differentiate into melanocytes upon reaching the skin [6]. There are many reports of familial aggregation of congenital nevi.

One study found that the MC1R (melanocortin-1-receptor) genotype, which corresponds to a red-haired genotype and a tendency to increased birthweight, was overrepresented in a cohort of congenital melanocytic nevi affected Northern European patients. How MC1R variants promote growth of congenital melanocytic nevi and the fetus itself is unknown as is the application of this finding to non-european and more darkly pigmented races [7].

Congenital nevi have been stratified into 3 groups according to size. Small nevi are less than $1.5 \mathrm{~cm}$ in greatest diameter, medium nevi are 1.5-19.9 cm in greatest diameter, and large or giant nevi are greater than $20 \mathrm{~cm}$. Giant nevi are often surrounded by several smaller satellite nevi.

Large congenital nevi of the head or posterior midline may also be seen as a component of neurocutaneous disorder, with cranial and/or leptomeningeal melanosis. Neurocutaneous melanosis may result from an error in the morphogenesis of the neuroectoderm, which gives rise to the melanotic cells of both the skin and meninges. Clinically, patients may present with 
increased intracranial pressure due to hydrocephalus or a mass lesion. The prognosis of patients with symptomatic neurocutaneous melanosis is very poor, even in the absence of malignancy [8]. Significant association is between giant congenital nevi and neurofibromatosis, with development of neurofibromas [9]. The histology is characterized by the presence of melanocytes in the epidermis ordered in theques and/or malanocytes in the dermis as sheets, nests, cords and/or single cells [10].

The histology of large congenital nevus may be delivered into nevus cell, neuroid, epithelioid cell and/or spindle cell, dermal melanocytic and mixed [11].

In the nevus cell type histology may appear identical to acquired nevi, but in the congenital nevus melanocytes are more in the lower two-thirds of the reticular dermis or deeper and more associated with neurovascular structures in the reticular dermis. In the neuroid type of giant congenital nevi, the dermis imelanocyte cells appear to be arranged in palisaded around a cellular mass of homogeneous material (Varocay Body) and sheating of nerves by neuroid tissue (neuroid tubes). The neuroid type of giant congenital nevus may be associated with congenital anomalies of bone (club foot, spina bifida, atrophy). In the Spindle cell and/or epithelioid cell type of giant congenital nevus, the dermis is infiltrated in whole or in part by nests or sheets of epithelioid and/or spindle cells, but unlike acquired variety is involved deeper the reticular dermis, with neuroid elements. Sometime, in giant congenital nevi, architectural and cellular features may be so atypical making differentiation with melanoma very difficult. In the dermal melanocytic type of giant congenital nevi, appearance may be that of a giant blue nevus.

In large congenital nevi are occasionally present, within melanocytes, trace of other tissue like muscle, bone, placenta. Other tissues occasionally present intermixed with melanocytic elements are hemangiomas, increased numbers of mast cells, cartilage, calcificacion. Associated tumors include schwannoma, neuroid tumors, lipoma, rhabdomyosarcoma, neurofibroma, sebaceous nevus, blue nevus, hemangioma, lymphangioma and mastocytoma, nevi of Ota and Ito, Spitz nevus [12]. The etiology of congenital melanocytic nevi has not been elucidated. One possible cause is a mutation. An association between infantile hemangiomas and congenital melanocytic nevi has been suggested [13]. Future investigation may yield more definitive causative factors. A review of dermoscopy patterns in congenital nevi found that most nevi demonstrate a reticular, globular, or reticuloglobular pattern. The findings varied with age and the anatomic location of the nevus, with the globular pattern found more often in younger children and the reticular pattern found in patients aged 12 years or older [14]. The role of dermoscopy in congenital nevi is currently recruiting.

\subsection{Association between congenital nevi and melanoma}

The risk of melanoma development is proportional to to the size of congenital nevus., with a clear evidence of increased risk in patients with congenital nevi involving over $5 \%$ of the body surface. For giant congenital melanocytic nevi, the risk of developing melanoma has been reported to be as high as 5-7\% [15]. Risk for the development of melanoma in smaller nevi has not been well quantified and the matter is still controversial (Fig.1). Also suggested is that melanoma developing within smaller congenital nevi usually occurs at puberty or later and 
develops more superficially in the skin, where it is easier to detect clinically. The lifetime risk of melanoma for patients with very large congenital nevi has benn estimated, approximately and considering variations in several countries and studies, at least $6 \%$.

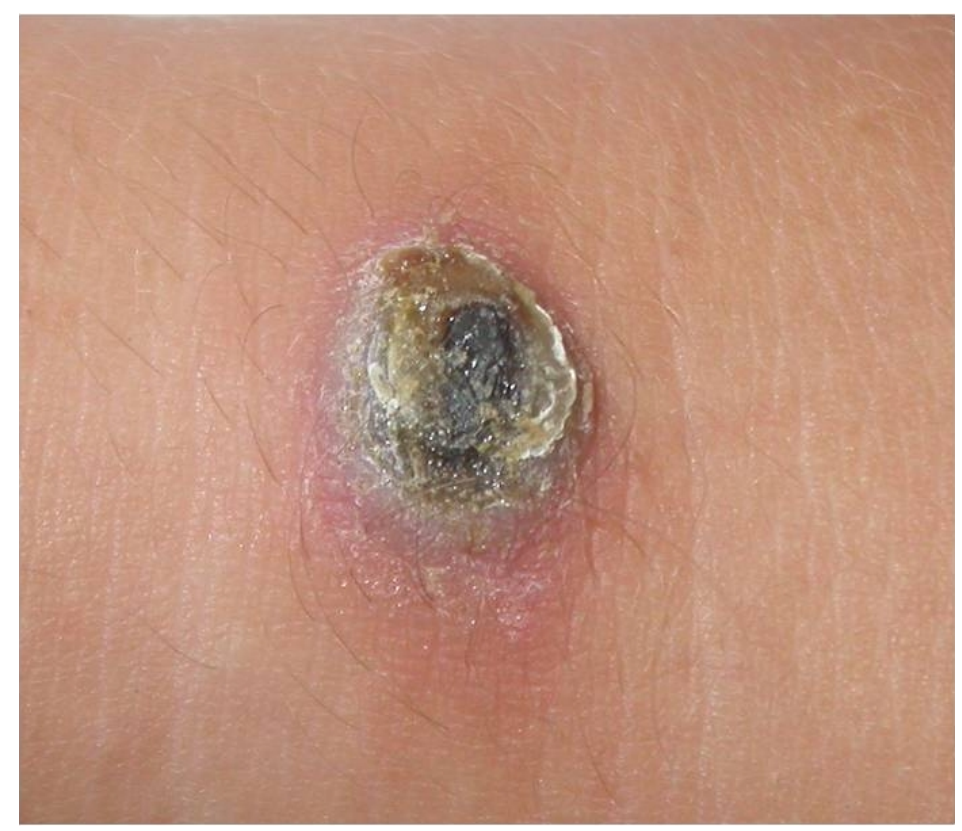

Figure 1. Malignant melanoma diagnosed in a small congenital lesion of the dorsal foot

\subsection{Spitz nevus}

It is also defined by other terms, including epithelioid cell and/or Spindle cell nevus, juvenile melanoma, benign juvenile melanoma. It occurs normally in children, but may appear in 15\% of adolescents and adults. Spitz nevus is a unique, acquired, usually benign melanocytic tumor, so alarming in its clinical presentation and sometimes histologically confused with melanoma. It is possible that some lesions regress spontaneously. It can appear pink or tan, as a papule, often with teleangiectasies on the surface (Fig.2). A variety called Reed nevus, more frequent In adults, may also be confused with melanoma, but histologically is an acquired, predominantly spindle cells variety, darkly pigmented [16].

\subsection{Common acquired melanocytic nevus}

Acquired melanocytic nevus is a common disorder of melanocytes, occurring as a pigmented benign lesion, possibly localized in every part of the skin (palmoplantar areas included) and 


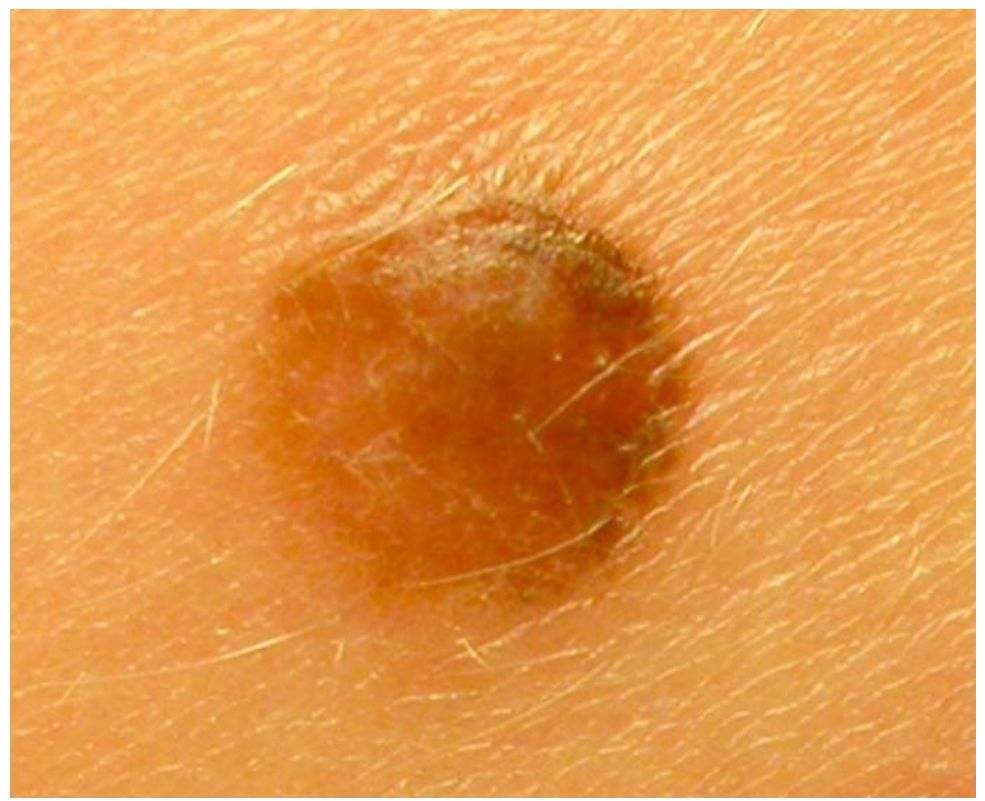

Figure 2. Spitz nevus

oral, ocular, genital mucosae. They first can appear after 6-12 months of life. Histology classifies acquired melanocytic nevi as a collection of melanocytic cells in the epidermis (Junctional), dermis (Intradermal), or both (Compound), disposed in isolated elements (epidermal variety, lentiginous pattern) or aggregated (junctional, intradermal and compound variety). There is evidence that number and size of common acquired nevi is associated with familiarity. Studies documented an increased number of nevi for pale skin, blond hair, blue or green eyes, tendency to sunburn. Typical acquired nevi usually have a round or oval, symmetric shape and relatively well-demarcated, smooth borders. The surface of nevi may be flat-topped, dome-shaped, papillomatous or peduncolated. More elevated acquired nevi tend to be more lightly pigmented, and flatter acquired nevi tend to be more darkly pigmented. More elevated and less pigmented lesions tend to have a prominent intradermal melanocytic component, whereas flatter and darker lesions have a more prominent junctional melanocytic component and a less prominent dermal component. Changes in acquired melanocytic nevi can be physiologic in puberty, pregnancy, corticosteroid administration and sun exposure; also changes may occur slowly during the years as normal evolution of nevi. Though most of times changes are benign, in presence of alterations of symmetry, color, borders, extension or regression, especially in a short time (months), a periodic monitoring of all nevi on the skin and mucosa is necessary, preferably with dermoscopy. When melanoma occurs on a melanocytic acquired nevi, changes may be global or, most of times, partial, that is the reason why asymmetry is a predominant parameter to be evaluated [17]. 


\subsection{Blue nevus}

The blue nevus consists of an acquired or congenital blue, blue-gray or blue-black papule, plaque or nodule, histologically composed by dermal dendritic, fibroblast-like cells containing melanin. Most of times it is localized on dorsa of hands and feet, usually singular. Common blue nevi remain unchanged or possibly regress. Particular types of blue nevi are:

- Cellular blue nevus is a blue-gray nodule or plaque 1 to $3 \mathrm{~cm}$ diameter mostly located on the buttock or sacrum.

- Combined blue nevus-melanocytic nevus, sometimes confused with atypical nevi or melanoma.

Malignant blue nevus, may develop in contiguity with cellular blue nevus, nevus of Ota, or de novo [18].

\subsection{Dysplastic melanocytic nevi}

Dysplastic nevus is an acquired, usually atypical-appearing melanocytic tumor, characterized histologically by epidermic and/or dermal melanocytic dysplasia. Dysplasia refers to abnormal tissue development. When applied to melanocytic tumors, dysplasia is referred to a disordered melanocytic proliferation in association with discontinuous and variable cellular atypia (mild, moderate and severe). About this spectrum of atypia, from slight to marked may be said that intraepidermal melanocytes in dysplastic melanocytic nevi occupy an intermediate position between typical and malignant, basing on nuclear and cytoplasmic features. Not all atypicalappearing melanocytic lesions have an atypical histology. It is generally believed that a melanocytic nevus appearing asymmetric, irregular in borders and pigmentation and with a diameter equal or more than $6 \mathrm{~mm}$ is considered dysplastic, but these characteristics are referred to "Atypical acquired Nevus", also called "Clark Nevus". Although the diagnosis of dysplastic nevus is suspected because of the atypical appearing, histological confirmation is required to establish the presence or not of dysplasia. It is important to define if atypical nevus is dysplastic, because it is a potential histogenic precursor of melanoma and marker of increased melanoma risk.

\subsection{Halo nevi}

Halo nevus, also referred as "Sutton's nevus" or "leukoderma acquisitum centrifugum", is a nevus surrounded by a macule of leukoderma (hypopigmented or apigmented area). It occurs in up to $1 \%$ of general population, with a peak of incidence in the second decade. It is commonly composed of a central pigmented nevus and an acquired surrounding depigmented halo. From 25 to $50 \%$ of patients have more than one halo nevi. Nevus regression can be complete and is caused by a lymphocytic aggression against nevus melanocytes with involvement of surrounding epidermal melanocytes. Association with vitiligo needs clinical and anamnestic analysis as a history for melanoma. 


\subsection{Nevus of Ota and Ito}

Ota first described this nevus and called it "nevus fuscocaeruleus ophtalmomaxillaris. Nevus of Ota is usually congenital but may appear in early childhood or in puberty. It is usually characterized by unilateral, flat, blue-black macules in the skin innervated by the first and second branches of the trigeminal nerve. Oral, nasal and pharingeal mucosae, conjunctivae and tympanic membranes may be involved. More rarely pigmentation may extend to cornea, optic nerve, fundus oculi, retrobulbar fat and periosteum. Enlargement and darkening may be observed over time. Histology shows stellate melanocytes widely scattered in the reticular dermis. Overlying melanocytes may be reduced in size and contain increased melanin. Nevus of Ota does not improve with time. 66 cases of melanoma development in nevus of Ota have been reported. Effective treatment is photothermolysis with Q-Switched LASER needing multiple sessions, with good results. Nevus of Ito is analogous to nevus of Ota and may coexist in the same patient. The difference between the two types of nevi is that nevus of Ito involves the distribution of the lateral supraclavicular and brachial nerves.

\subsection{Melanoma}

Melanoma is a malignant tumor resulting from the trasformation of melanocytes of the skin and less frequently of mucosae. During embryonic life, melanoblasts migrate from neural crest to the basal-cell layer of epidermis and a minus part to skin appendages and dermis. Melanoma can arise from melanocytes located in these sites.

Risk for the development of melanoma remains low in pre-pubertal age, with an annual incidence of 0.7 cases per million children aged 0-9 years. Reaching adolescence the incidence of melanoma increases, with a rate of 13.2 cases per million children aged 15-19 years [2]. Prevention and early recognition of melanoma is mainly applied to adults through periodic clinical and dermoscopic controls. Only in recent years data documented alarm about increasing incidence of melanoma in adolescents. This increase, combined with other data about congenital nevi, allow physicians in the last years to play a crucial role in the identification of children at risk for melanoma, with particular regard to detection of risk factors in children and adolescents, and education about sun and artificial ultraviolet exposure.

\section{Types of primary melanoma of the skin}

\subsection{Lentigo maligna}

Lentigo maligna is a precursor lesion that may progress into invasive melanoma. It appears as a macular, freckled-like lesion of irregular shape, occurring most often in elderly patients (age over 60 years) in sun exposed and sun-damaged, atrophic skin. Lentigo maligna normally grows slowly for long periods (years) with a prolonged radial growth phase, beforeevolution in Lentigo Maligna Melanoma. Hstopathology reveals atrophic epidermis and increased numbers of atypical basilar melanocytes that may extend down the hair follicles and skin appendages. 


\subsection{Lentigo maligna melanoma}

It is a melanoma in situ slow growing progressing to invasive melanoma with nests of malignant melanocytes invading the dermis. Lentigo Maligna Melanoma represents 4 to $15 \%$ of all melanoma.

\subsection{Superficial spreading melanoma}

SSM is the most frequent type of melanocytic malignancy representing $70 \%$ of all melanomas.

Most commonly it occurs on the upper back of men and on the legs of women, although it can develop at any site, mucosa included. The usual history is that of slow change (months to 1-5 years) of a preexisting melanocytic lesion or can arise "de novo".

SSM most frequently presents as a macule asymmetric, variegated pigmentation (from brown to black, with variable presence of blue-gray, gray-white or pink areas as sign of regression along the borders or inside the lesion. Borders may present intact initially in presence of a precursor melanocytic nevus, but mostly are irregular. Dermoscopy can reveal better all these alterations, and more other parameters typical of SSM (salt-peppering areas, star-bust aspect, pseudopodes at borders, blue-white veil and so on). Diagnosis of SSM may be done from a macroscopic view, but dermoscopy helps much for confirmation diagnosis and for early diagnosis, when it is difficult to find macroscopically some alterations typical of melanoma.

Histopathology reveals a "pagetoid" distribution of atypical large melanocytes thruoughout the epidermis. The large cells may occur singularly or in nests and have a monomorphous appearance. In the dermis areas of invasion of atypical melanocytes are present.

\subsection{Nodular melanoma}

The second most common subtype of melanoma is nodular melanoma with a frequency of 15 to 30 percent of all types. NM is remarkable for its rapid evolution and may arise from melanocytic nevi or normal skin (de novo), but in lack of an apparent radial growth phase. NM is more common to arise "de novo" than from a preexisting nevus.

NM appears typically as a blue-black, blue-red or amelanotic nodule or papule. In certain cases it may be difficult to diagnose especially when it appears as an amelanotic reddish lesion.

Histopathology may demonstrates a little tendency for intraepidermal growth, but it typically arises at dermal-epidermal junction and from its onset with extention to the dermis, composed of large epithelioid cells, spindle cells, small cells, or a mix of these different cells.

\subsection{Acral lentiginous melanoma}

ALM is more common in darker skin individuals (60 to 72 percent in blacks, 29 to 46 percent in asians). In white skin people it represents only 2 to 8 percent of melanomas. ALM occurs on palms and more often on soles, or beneath the nail plate.

The biologic behavior of ALM is traditionally considered more aggressive with a poorer prognosis and this may be due to late diagnosis and/or to the different biologic origin of it. 


\subsection{Melanoma of the mucosa}

It involves oral, nasal, vulva, anorectal, conjunctival mucosae and it may occur with or without a radial growth phase.

\subsection{Desmoplastic melanoma}

It is a rare subtype of melanoma, locally aggressive and with high rates of local recurrence. It may arise in association with LM, ALM and mucosal melanoma or "de novo". DM may appear as a pigmented macule, papule, nodule or reddish. Histologically it is caracterized by fibrous tissue and atypical spindle-shaped melanocytes that show a propensity to infiltrate cutaneous nerves.

The incidence of malignant melanoma is rapidly increasing in the last decades. The surveillance, epidemiology and results program (SEER) has documented a 32,7\% increased in mortality rates over the period 1973 to 1995 . on the other hand, the overall survival rate has been improving for melanoma in the last decades. Currently, cutaneous melanoma accounts for approximatively $1 \%$ of all cancer deaths [19, 20, 21, 22].

More recent studies indicate that the rate of increase in all age groups was $2.8 \%$ per year from 1981 to 2001 and in children (age < 20 years) was 1.1\% per year from 1975 to 2001.1 The diagnosis is often delayed in children since melanoma is rare (300 to 420 new cases per year), and benign lesions, especially Spitz nevus, may mimic melanoma. The prognosis is good when there is prompt identification and wide local excision of early disease, but poor for those with advanced disease at presentation [23]. Case-control studies in adults have identified multiple host and environmental factors associated with increased risk of malignant melanoma. Host factors include fair skin, white race, blond or red hair, light eye color, tendency to burn with UV radiation exposure, increased number of benign nevi, dysplastic nevi, family history of melanoma, and xeroderma pigmentosum.6,7 Environmental factors include sunburns, often as a child, and increased exposure to UV radiation. Proposed risk factors for paediatric melanoma include congenital, dysplastic, or increased number of nevi; inability to tan; blue eyes; facial freckling; family history of melanoma; disorders of DNA excision repair like xeroderma pigmentosum; acquired or congenital immunosuppression and a previous history of malignancy [24, 25, 26].

Analysis regarding children and young adults with melanoma between 1973 and 2001 show how older age, more recent year of diagnosis, female sex, white race, and increased environmental UV radiation were all associated with a significant increase in the risk of melanoma. In the first year of life, the incidence of melanoma is similar by race, but it diverges by age 5 to 9 years and is more than 40 -fold higher in white individuals by age 20 to 24 years.

The increase in incidence of melanoma in children, especially in adolescents, is similar to that seen in young adults. This may reflect increased cumulative UV exposure during childhood or adolescence, greater awareness and more frequent diagnosis of melanoma (eg, versus atypical Spitz nevus), differences in genetic predisposition, and/or other environmental factors. The increased risk of melanoma in girls, particularly on the lower extremities, may be a result of increased UV exposure. In adults, sun-related behaviours differ between men and 
women. [22] The increased rates of melanoma in adolescent and young women may reflect sunbathing or the widespread (> 25\%) practice of indoor tanning [28].

Prognosis for young children, adolescents, and young adults with melanoma appears to be similar. Also increased is the risk of death in male children, those with regional or distant metastasis, primary sites other than the extremities or torso, increasing thickness of the primary lesion, earlier year of diagnosis, and previous cancer.

Melanoma-specific survival in children has improved by approximately $4 \%$ per year during the last 3 decades. It is difficult to explain this dramatic improvement. Although earlier diagnosis could be associated with improved survival, there has been no decrease in lesion thickness over the last decade. Furthermore, survival has improved for all stages of paediatric melanoma. The most notable improvement has been in the "unstaged" group, likely due to more complete staging. There are important differences in young children (age $<10$ years) with melanoma compared with adolescents and young adults that may reflect distinct tumor biology and/or host characteristics.

Published large series of paediatric melanoma report 5-year survival rates of $74 \%$ to $80 \%$. This is significantly worse than the $91 \% 5$-year overall survival seen in recent analysis, after the exclusion of cases of melanoma in situ [29, 30]. Accepted prognostic factors in adult melanoma include primary lesion thickness, ulceration, and non-extremity site; increased age; regional lymph node involvement; satellite or in-transit metastases; elevated serum lactate dehydrogenase level; visceral or brain metastases.[4,9 However, prognosis and prognostic factors in children are less defined. In a recent review of more than 300 cases, the outcome for paediatric patients (5-year survival of $74 \%$ ) was slightly worse than that of young adults, but these survival estimates have limitations. In a large European registry study of children, male sex, unfavorable site (lesions on the trunk), and/or second primary or regional or distant metastasis. [10] Advanced stage has been associated with poor prognosis in other paediatric studies [27].

In summary, paediatric melanoma is an important and increasing problem. Factors conferring risk of adult melanoma, including older age, white race (blue eyes, blond or red hair, freckling tendency, liability to tan and tendence to sunburn), family history of melanoma, elevate number of acquired melanocytic nevi (double risk in 50 to 99 of acquired melanocytic nevi), dysplastic nevi, environmental exposure to UV radiation, congenital or acquired immunosuppression are also important in paediatric melanoma.

\section{Indications for excision of nevi}

Indication for excision are usually assessed by dermatologists, paediatric surgeons, paediatrician and physicians.

Nevi can be divided into congenital and acquired. In turn congenital nevi can be small, intermediate and large $(>20 \mathrm{~cm})$. Melanoma risk in small congenital nevi is debated, although in some reports it seems to be of importance [32] Some studies demonstrated an increased risk of malignant melanoma in small lesions as well as in intermediate lesions, but this is still a 
matter of controversy. In the lack of consensus about systematic removal of small congenital nevi, careful dermatologic monitoring and prompt excision after clinical changing is recommended [33] In the Literature there is a large agreement on the excision of large congenital nevi. In fact they show an increased risk for development of malignant melanoma, varying from $1 \%$ to $31 \%$. Nonetheless malignancy is reported to occur despite complete excision and may be not preventable [34, 35]

Indications for surgical excision [36] of acquired nevi are mainly related to lesions resembling malignant melanoma, such as atypical nevi, Spitz nevi, and lesions presenting clinical signs and symptoms as diameter of more than $5 \mathrm{~mm}$ or increasing diameter, irregular margins, border notching, irregular pigmentation, asymmetry, rapid onset or increase in diameter, ulceration, bleeding, pain and itching. These last signs seem to represent the presentation symptoms of malignant melanoma in $85 \%$ of cases [37] Although the risk of malignant melanoma is higher in familial atypical nevi rather than in nonfamilial, features of atypical nevi have been reported to be the most frequent indication to surgery [38]

Some special consideration is given to Spitz nevus for possible misdiagnosis with MM. Most dermatologists and physicians recommend biopsy. In an interview with dermatologists, most responding specialists (93\%) recommended the biopsy of suspected Spitz nevi. Sixty-nine percent of physicians would completely excise a lesion that was histologically diagnosed as an incompletely removed Spitz nevus. Seventy percent of general dermatologists and $80 \%$ of pediatric dermatologists would recommend excision with a 1- to 2-mm margin of normalappearing skin around a Spitz nevus [39]. The lack of consensus about the nature and the ideal management of Spitz nevus reflects the uncertainty in histopathologic distinction between Spitz nevus and melanoma, and such a concern influences management. By some authors 2 $\mathrm{mm}$ margins excisional biopsy of clinically appearing Spitz nevus, as of any nevic lesion, is recommended

In some cases nevi located in sites of clinically difficult monitoring or placed in sites of exposure to frequent trauma are an indication for excision $[38,40]$

\section{Treatment of acquired nevi}

Acquired melanocytic nevi begin to appear after the first 6 months of life and increase in number during childhood and adolescence, typically reaching a peak count in the third decade and then slowly regressing with age. They are classified as junctional nevi if the nests of melanocytes are in the dermal - epidermal junction, intradermal nevi if the nests are in dermis and compound nevi if the nests are located in both sites.

They usually have a diameter of less than $6-8 \mathrm{~mm}$, a homogeneous surface, pigmentation, round or oval shape, regular outline, and demarcated border, sometimes with pigmentary stippling or perifollicular hypopigmentation.

These are rarely complicated by evolution in malignant melanoma so conservative treatment, that is clinical periodic control, is usually enough. 
However, when removal of a naevus is necessary we have to do some considerations especially in order to the characteristics of the nevus and its position.

\subsection{Anesthesia}

Indications and surgical techniques of the excision of a skin lesion are similar to the adult and so the actual difference with children is the anesthetic management.

Analgesics are commonly administered prior to surgical procedure in children. The concept of preemptive analgesia is still controversial and its effectiveness may depend on the type of surgery. Nowadays the most commonly used medications are acetaminophen alone or with codeine, but also non - steroidal anti - inflammatory medications (ibuprofen) are effective analgesics for perioperative pain, but less used for the impact on bleeding.

In association with analgesics and anesthetic medicament the use of sedative is very common and useful also in children as in the adult. Midazolam is very convenient in children because not only alleviates the anxiety of surgery, but also induces an anterograde amnesia, useful for treatments requiring multiple visits. Furthermore, it can be administrated in various vehicles via the nasal, rectal, sublingual that are less traumatic than intramuscular injection in children.

Fentanyl is 100 times more potent than morphine and has less influence on GI motility than morphine, so reducing the effect of emesis and oxygen desaturation becoming more tolerable in children.

General anaesthesia is used in the excisional surgery of large lesions and occasionally in non - cooperative children. It is a very safe procedure, but the rate of complication increases in case of surgery in the first year of life and when the anesthesiologist has no pediatric experience.

In recent years the use of topical anesthetics, especially EMLA cream (eutectic mixture of local anesthetics), knows an important development in office procedures in children, although the incorrect use of EMLA causes failure in reducing pain of dermatologic procedure. In particular for an effective absorption of the anesthetic into the skin an occlusive dressing is useful.

\subsection{Surgery}

Any skin lesion presenting features of malignant melanoma are biopsied. Although melanoma in children is rare, the most frequent indication for excision of an acquired nevus is early diagnostic evaluation because of melanoma concern.

Excisional biopsy is the treatment of choice. It may be elliptical, wedge or circular, but the first is the most commonly used.

\subsubsection{Simple excision}

Skin incisions should be planned along or parallel to the relaxed skin tension lines (RSTLs). This allows, on one hand, a better wound healing, an easier matching of edge of the wound and lower tension of the sutures and, on the other hand, to hide the wound in a skin fold. 
The elliptical excisional biopsy, as the other type of excisional biopsy, must include a portion of healthy tissue of $0,2 \mathrm{~cm}$ wideness from the perimeter of the lesion and the subcutaneous tissue. The possible exuberant tissue, called "dog - ear" can be corrected by extending the ellipse or removing the excess skin with a $\mathrm{L}$ or $\mathrm{Y}$ incision.

The wedge excisional biopsy is usually utilized for the lesions located on or close to the free margin of some particular structures as eyelid, lip, nose and ear. In the eyelids it is possible to make an incision along the edge and remove only the skin, although some lesions require excision of full thickness eyelid. $1 / 4$ of eyelid or $1 / 3$ of the lower lip can be completely excised and the defect closed with a simple suture without elaborated reconstructions.

The circular excisional biopsy is used when a skin limited incision is needed as in the case of nose or in the anterior region of the auricle. The defect can be closed with a skin graft or a skin flap.

When repairing the loss of substance by combination of skin edges is impossible, other techniques are necessary:

Grafts, rarely used for the excision of acquired nevi

\subsubsection{Flaps}

A flap is a portion of one or more tissues transferred from a donor site to a receiver maintaining a neurovascular connection ("pedicle").

The use of flap has many advantages including that of allow the repair of the defect by means of tissue similar or equal to those of the receiving site. It is very important for specialized tissues as lip or eyelid.

The skin flap, composed by skin only or including subcutaneous tissue, is transferred from one part of the body to another with its neurovascular pedicle or attached by just a margin to preserve vascular support.

There are two types of skin flap: those which rotate around a pivot point (rotation, transposition and interpolation flaps) and advancement flaps (single pedicle, V - Y, Y - V and bi pedunculated.

The rotation flap is a semicircular flap that rotates from its pivot point to the receiving site (Fig.3). If tension is too high, the incision can be extended by a reverse incision from pivot point along the base of the flap (backcut).

The transposition flap is composed by a rectangle or square of skin and subcutaneous tissue that rotate around a pivot point close to lesion.

The advancement flap is brought forward on the lesion without rotation.

This can have only one pedicle, so feeding is maintained by exploiting the elasticity of the skin.

The advancement $\mathrm{V}-\mathrm{Y}$ flap more than a flap is a V incision whose sides are closed in such a way that the final suture gives a $Y$. 


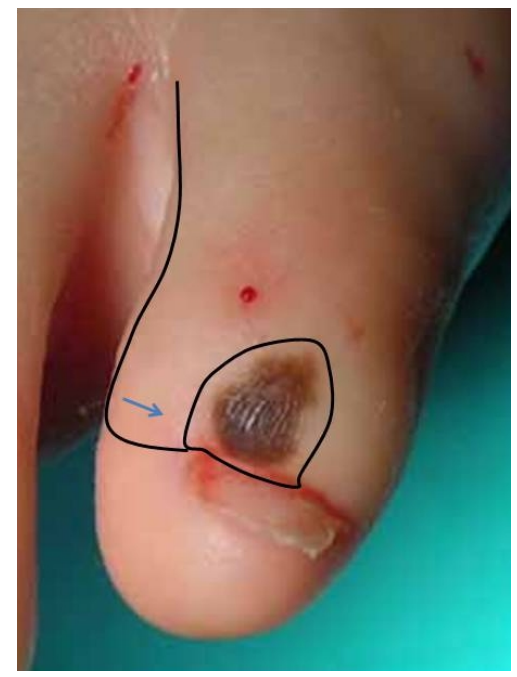

Figure 3. Rotational flap to excise a peliungueal nevus

\section{Treatment of congenital nevi in the pediatric age}

\subsection{Indications and timing}

Surgery of congenital nevi is predominantly indicated for preventive reasons, related to the risk of developing a malignant melanoma within the lesion during life. This indication has been discussed more extensively elsewhere in the chapter. Evaluation of all small and medium $\mathrm{CNN}$ for prophylactic excision should take place before the patient is aged 12 years. After this age, malignant potential rises sharply. Some authors advocate prophylactic excision of all CNN $[35,41,42]$,

whereas others advocate clinical monitoring of small [38] or both small and medium nevi [43] The incidence of malignant melanoma appears higher in large congenital nevi, in the scalp, back, and buttocks and requires removal first. This increase in incidence is likely secondary to the total body surface area. The presence of an enlarging nodular mass indicates malignant change and requires immediate treatment. This mass may represent a rare neuroectodermal sarcoma.

The other important indication to treatment is the possible discomfort due to the presence of a visible difference or even disfigurement. Psychological implications of this condition can involve both parents and children, in a different manner for different ages. Parents feelings about their child's appearance are likely to influence the child's perception of his or her disfigurement, the developing body image and feelings of self-worth [44]. Parental strategies to deal with a physical difference vary considerably. Some discuss it openly, others may act as if it does not exist, parents may be over-protective or children may avoid issues related to their appearance for fear of upsetting their parents 45 . Most children with a visible difference 
often experience appearance-related teasing and bullying during the course of their school career [46]. A link between appearance-related teasing, body dissatisfaction and general psychological disturbance has been discussed by Gilbert and Thompson [47]. The physical and psychological changes associated with adolescence increase the importance of physical appearance, and having a disfigurement during this period may present particular challenges. Image counts in the dating game, and joining an acceptable social grouping can be difficult if social confidence has been in some way weakened. Harter [48] reported that teenagers who believed their appearance determined their self-worth had lower self-esteem and greater depression than adolescents who believed their self-worth determined their feelings about their appearance.

Nonetheless, indications to surgical treatment should be carefully evaluated when the motivation of patient is doubtful. Treatment decisions made during childhood, adolescence and adulthood can be stressful. Deciding whether or not to undergo appearance-altering surgery may not be easily accepted, and those affected can question any motivation for putting themselves through the associated stress [49] Furthermore, expectations about outcomes may be unrealistic, and can generate disappointment when the aesthetic result becomes apparent. Given the multifaceted nature of surgical adjustment, the prevailing model of care needs to be expanded to offer psychosocial support and intervention as routine adjuncts or alternatives to surgical treatment. Interventions need to be carefully planned to take account of individual physical (e.g. growth) and social issues and the child with a visible difference or disfiguring lesion should receive continuing psychological support, preoperative assessment and follow up during the course of treatment.

Surgical excision with reconstruction, is the mainstay of treatment. If direct closure after complete excision is not possible, reconstruction may include excision with skin grafts, skin flaps, tissue expansion with subsequent flap rotation or full thickness skin grafting, autologous cultured human epithelium, artificial skin replacement, and free tissue transfer after tissue expansion [50, 51, 52] Chemical peels, dermabrasion, and laser treatments are adjunctive treatment choices, that have not been demonstrated to decrease the malignant potential, because of incomplete removal of cells in the treated area. If surgical excision is not feasible, management consists of examination and high-quality photographic documentation for life.

Serial excision of large congenital nevi by skin expansion should preferably start in early months of life, for their malignant potential and for their size, requiring many surgical stages. It is usually addressed at age 6 months, to decrease anesthetic and surgical risks [52, 53, 54]. Attempts to complete the treatment of particularly disfiguring lesions is preferably carried out before age 5, when possible, or at least in the pre-adolescent, to prevent important psychosocial implications linked to the different developmental stages of the child and to parental behavior [55]. The goals of treatment are to remove all or as much as feasible of the CNN and reconstruct the defect, preserving function and maintaining the aesthetic appearance. Each case requires tailoring of the operations to fit the anatomic defect and to respect anatomic units and relaxed skin tension lines when possible. Excision begins in the 6-9 month range, placing procedures 3-6 months apart. 


\subsection{Surgical techniques}

Technical choices vary depending on nevus size and anatomical site and may be challenging in some cases of giant nevi.

General considerations, including anatomical and surgical principles, should be remembered. Incisions are planned according to the orientation of the relaxed skin tension lines (RSTL) when possible, with attention to the most favorable and less visible site of the resulting scar, especially when the use of skin expanders is required.

A variable amount of subcutaneous tissue should be included in the excision, for its diagnostic value in the rare occasional finding of melanoma in the specimen. This amount is thinner in facial areas, to avoid nerve injuries, but enough to include hair follicles.

\subsubsection{Small congenital nevi}

Excision of small congenital nevi (diameter $\leq 1.5 \mathrm{~cm}$ ) is usually performed with $2 \mathrm{~mm}$ margins of normally appearing skin, by simple excision. In special areas, as some parts of the nose, lip, eyelid or ear, serial excision or rotation, advancement or transposition flaps are often necessary.

\subsubsection{Medium size congenital nevi}

Excision of medium size nevi (diameter $>1.5<19 \mathrm{~cm}$ ) can be achieved by serial excision or tissue expansion.

\section{Serial excision}

The efficacy of serial excision for the treatment of medium size congenital nevi has been reported by different authors [56] and it is the indication of choice when the procedure can be easily planned in 2 stages [57].

In children this indication can be extended to larger lesions, requiring more than 2 stages, when considering the possibility of avoiding morbidity related to tissue expansion, longer operating time of every stage, multiple expanding percutaneous injections and poor compliance by the patient. When surgical planning suggests too many operations to complete the removal of the lesion, tissue expansion should be seriously considered as an alternative.

\section{Techniques of serial excision:}

A symmetric, fusiform ellipse is drawn within the lesion, parallel to the RSTLs and the margins are undermined enough to obtain a tension free suture (Fig. 4)

In the subsequent period the surrounding skin is going to stretch and and adapt, releasing tension on the scar. After a minimum of 3 months a second excision is performed to complete nevus removal. 


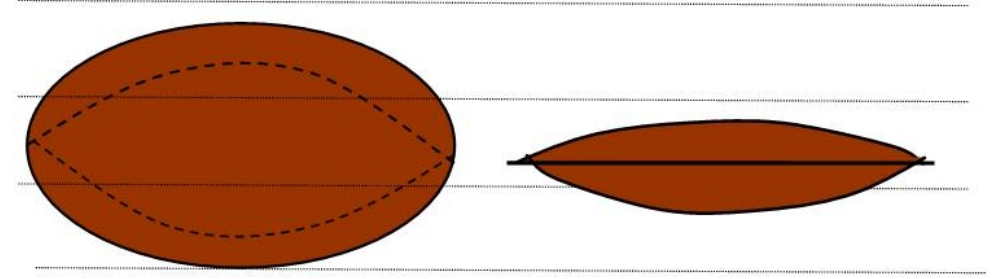

Figure 4. First stage of serial excision with the long axis of the lesion parallel tothe RSTL
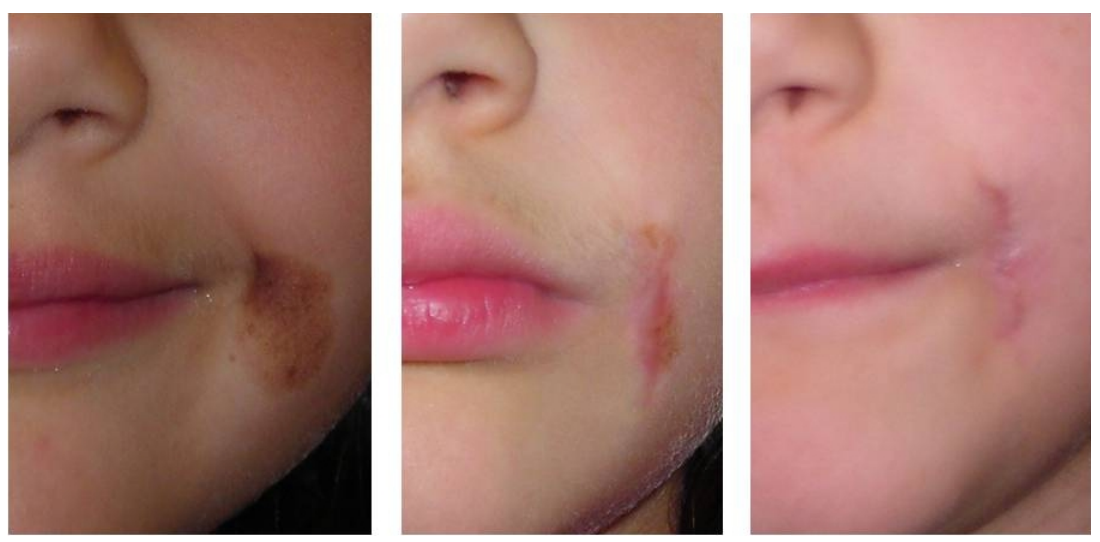

Figure 5. Serial excision of medium size congenital nevus, with final positioning of the scar in the nasogenien crease

When the resulting scar is desired to fall in a crease or for the treatment of particular anatomical sites, as nasal ala, oral commissure, lateral canthus, some modifications may be required. The fusiform excision may be planned to be eccentric and the skin undermined more on one side, to move the tissue in one direction rather than the opposite one. The direction of the prevalent movement can be towards a natural crease, the border of an aesthetic unit or an anatomical area not to be distorted, as nasal ala or oral commissure (Fig.5)

\section{Flap surgery}

In difficult anatomical sites and for wider intermediate sized lesions flap surgery is also indicated to obtain excision without tissue loss or distortion. An advancement flap may be used when its incision lines can be drawn along the borders of different aesthetic units. For example, in the case of a round nevus on the lateral aspect of nasal pyramid, incisions of an advancement flap could be outlined on the infraorbital and nasogenien folds to hide scars in 

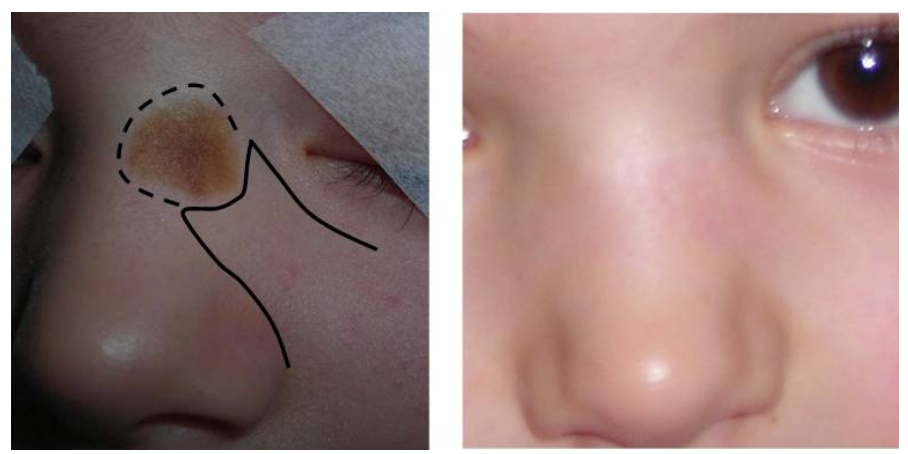

Figure 6. An advancement flap is drawn in the infraorbital area along infraorbital and nasogenien creases.
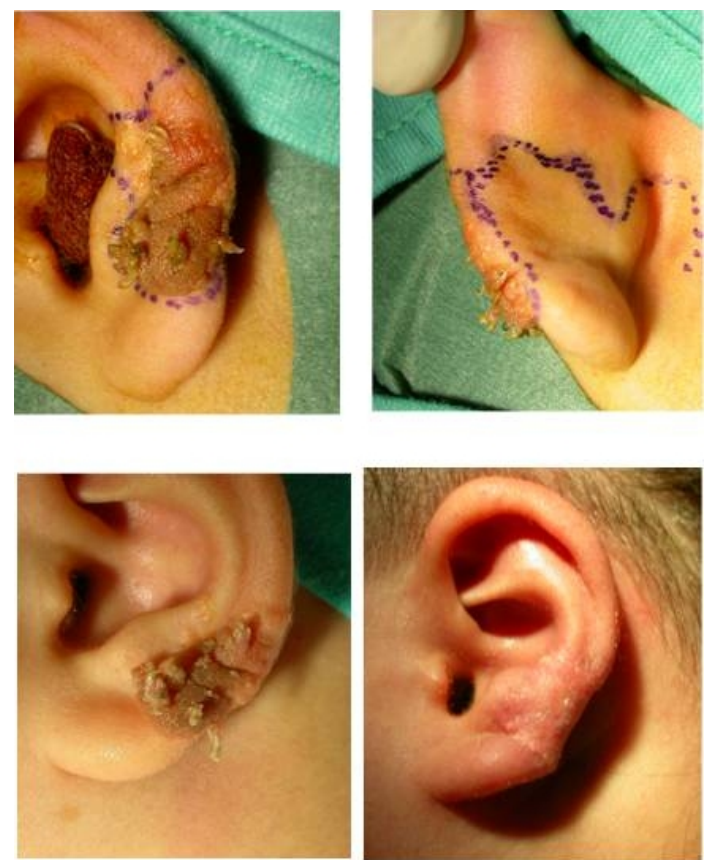

Figure 7. A bilobed flap is transposed from the retroauricular crease.

these creases (Fig.6). On the auricle, due to the adherence of local skin, a sufficient amount of tissue can be obtained from the retroauricular fold by a transposition flap (Fig.7).

\subsubsection{Large or giant congenital nevi}

Treatment of large or giant congenital melanocytic nevi (CGMN) (diameter $>19 \mathrm{~cm}$ ) always requires multiple surgical stages and complex strategies. 


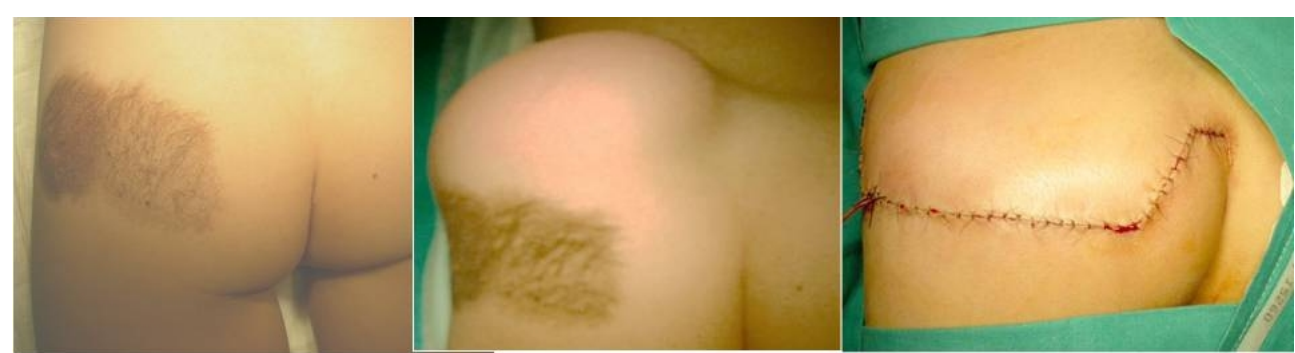

Figure 8. Skin expansion to excise a large congenital nevus of the gluteal region

\subsubsection{Tissue expansion technique}

Although multistaged direct excision, described elsewhere about medium size nevi, is sometimes feasible for the treatment of large lesions, skin expansion is the treatment of choice and will be discussed in more detail. In general terms, expansion of tissue is used to improve rotation, transposition or advancement of local or regional flaps, or to increase the harvest of full-thickness skin grafts. In adults, aside from their use in breast reconstruction, tissue expanders are used primarily for secondary burn and trauma reconstruction in the head and neck region. In the pediatric population, expanders have been used in a variety of reconstructive procedures. The most common indication in children is to reconstruct defects left by excision of giant congenital nevi (Fig.8).

Tissue expansion is contraindicated in infected skin. Although expansion is possible in radiated or scarred tissue, it is associated with a much higher complication rate and should be avoided whenever possible.

Surgical technique of skin expansion relies on the ability of skin and soft tissues to grow by generation of new tissue in response to tension. Tensive impulse is generated by implanting a subcutaneous device (expander) that is inflated over a period of weeks; new tissue is generated in response to the constant stretch caused by the progressive inflation. An increase in skin surface area after expansion is due to generation of new tissue rather than the stretching of existing skin, as supported by numerous studies. Fibroblast and epidermal hyperplasia induced by mechanical stress have been observed in culture. Histological response to expansion is similar in adult and pediatric skin. Within 1-3 weeks of expansion, the epidermis begins to thicken and the dermis thins while skin appendages do not change. The subcutaneous fat atrophy. Cellular proliferation reduces the resting tension of the skin over time, enabling further expansion to take place. Once the process is complete, the expanded skin eventually returns to its baseline thickness. The vessels of the skin and subcutaneous tissue also resume their pre-expanded size and number [58, 59].

Expanders are available in a variety of shapes and sizes, and there is no absolute ideal expander for a given site or condition. Expanders have different types of filling ports. These can be internal to the expander or remote and connected by a tube of various length, that is usually adjustable by the surgeon. Most experienced surgeons recommend using remote ports. These should be placed away from the expander. Internal ports have both a higher failure rate and 
a greater incidence of accidental expander rupture. In children, the use of internal ports is associated with a higher rate of exposure of the expander due to the pressure exerted on the skin by the port. As a rule, expansion proceeds best when the expander rests on a firm base like the ribs or skull. When placed within the abdominal wall, for example, expansion tends to be tess predictable. The incisions for expander placement and the remote port should be placed where they will not interfere with later advancement or compromise the blood supply to the expanded tissue. If possible the incisions for expander placement are placed in the proposed area to be excised. Incisions are never placed parallel to the edges of the expander. This creates a situation that increases implant exposure, additional scar tissue outside the lesion, possible stretching of the scar and a delay in inflation of expander. Incisions should be radial or almost perpendicular to the expander or in the form of a $\mathrm{V}$ or $\mathrm{W}$

The broad base of the $\mathrm{V}$ or $\mathrm{W}$ should be directed toward the expander, thus facilitating implant insertion and inflation because the lines of tension are perpendicular to the wound. The open end of the $\mathrm{V}$ should be at least 2 to $3 \mathrm{~cm}$ from the pocket to accommodate expansion. In addition, a sigma (lazy $S$ ) incision can also be beneficial in instances where partial excision of some of the lesion might be helpful during the insertion phase. By this approach, partial excision can be done while an expander is placed. Once the wounds heal, the expander can then be inflated without worry because the end of the incision is almost radial to the expansion process [60]. The expander should be placed on top of the deep fascia (or subgaleal in the scalp), unless the plan is to incorporate muscle into the expanded flap. The pocket should always be larger than the base diameter of the expander. Blunt dissection in a single fascial plane is safest for preserving blood supply. Filling the expanders intraoperatively with sufficient saline to eliminate dead space can prevent postoperative bleeding and hematoma. An alternative to traditional prolonged expansion is immediate intraoperative expansion combined with broad undermining of the defect. In rapid expansion, the skin initially expands due to its elasticity and the displacement of interstitial fluid. Within minutes, the alignment of the collagen fibers changes due to the stretch. This process yields up to $20 \%$ more tissue for flap coverage. Intraoperative expansion is indicated for relatively small defects, such as in coverage of defects of the ear.

The rate of inflation is variable and largely based on surgeon preference. Patient comfort and signs of tissue perfusion, such as tension, color, and capillary refill, guide the filling rate. Filling is usually initiated 7-10 days postoperatively and performed once or twice a week, based on the above mentioned criteria and patient tolerance. The rate of expansion depends both on the body site as well as patient factors. Some skin is more amenable to expansion, and some patients can tolerate the discomfort better than others [61]. Tissue expansion should continue until the expanded area is larger than the defect, usually up to 2 months. As a general rule, the diameter of the expanded flap should be 2-3 times the diameter of the skin that is to be excised

Most surgeons overinflate tissue expanders beyond the manufacturer's recommended maximum capacity. Studies have demonstrated that significant overinflation is possible before weakening or rupturing.

The use of rotation and transposition flaps enables the transfer of tension from the tip of the flap more proximally to its base. A single or double back-cut can be performed prior to inset 
in order to gain extra length. The donor site should be closed in layers after the implant capsule is excised.

Scalp. Although tissue expansion does not increase the number of hair follicles, the size of the hair-bearing region can be doubled without a noticeable decrease in hair density. As such, tissue expansion may be used to reconstruct the scalp when removal of a medium or large nevus is needed. Expanders are most commonly placed in the occipital or posterior parietal regions. They are placed under the galea, superficial to the periosteum. It usually requires up to 12 weeks to complete the expansion in children. Radial scoring of the galea at the time of surgery can facilitate the process. Once the expansion is complete, flaps are advanced or transposed, based on named arteries of the scalp. It is important to orient flaps so that the correct direction of hair growth is maintained. Although galeal scoring or capuslotomy incisions can be useful, wide undermining is a safer method of recruiting tissue.

Forehead. The brow position is the most important structure to preserve during forehead expansion. When possible, two or more expanders are used with incisions hidden within the hairline.

Midforehead nevi are best treated using an expansion of bilateral normal forehead segments and medial advancement of the flaps, placing scars along the brow and at or posterior to the hairline. Hemiforehead nevi often require serial expansion of the uninvolved area of the forehead to reduce the need for a back-cut. Nevi of the supraorbital and temporal forehead can be treated with a transposition of the expanded normal skin medial to the nevus. When the temporal scalp is minimally involved with nevus, the parietal scalp can be expanded and advanced to create the new hairline. When the temporoparietal scalp is also involved with nevus, a combined advancement and transposition flap provides the proper hair direction for the temporal hairline and allows significantly greater movement of the expanded flap. Once the brow is significantly elevated on either the ipsilateral or contralateral side from the reconstruction, it can only be returned to the preoperative position with the interposition of additional, non-hair-bearing forehead skin. The largest expander possible beneath the uninvolved forehead skin should always be used, occasionally even carrying the expander under the lesion [62].

Face and Neck. The skin of the neck and face is relatively thin. Therefore, multiple expanders with smaller volumes are preferable to a single large expander. In general, however, a single larger expander is preferable to several smaller expanders. Careful planning is essential in determining where to place the expanders, and where incisions should be located in order to preserve aesthetic units, facial symmetry and matching skin color and to avoid distortion of the eyelids and oral commissure. The expander is usually placed above the platysma muscle to avoid risk of facial nerve injury and to keep the flap from being excessively bulky. The expanded flaps are positioned by advancement, rotation, or transposition. Incisions should be placed in skin creases such as the nasolabial fold or along the margins of aesthetic units. Expanding the hairless skin adjacent to the mastoid region can increase the available tissue for reconstructive procedures of the ear. The skin above the clavicle can be expanded to provide full-thickness skin grafts to the face. 
Trunk. Unlike the head and neck, there are very few critical landmarks on the trunk that must be preserved. Aside from the breast and nipple-areola complex, distortion of the skin and soft tissues of the trunk is well-tolerated. For defects requiring excision, multiple expanders surrounding the defect are often employed. Expanders can also be used to expand the skin of the abdomen for use as a donor site of full-thickness skin grafts.

Extremities. Tissue expansion in the extremities has been reported to have a higher complication rate, in comparison to other regions and therefore, especially in children, should not be a first choice. Blood supply and drainage of the extremities is inferior to that of the trunk and head. This predisposes limbs, especially below the knee, to an increased rate of wound complications such as infection, dehiscence and prosthesis extrusion. Multiple expanders are usually required in the extremites.

Complications. Among all patients, the major complication rate is about $10 \%$ and includes implant exposure, deflation, and wound dehiscence. Minor complications also occur in about $10 \%$ of patients. These include filling port problems, seroma, hematoma, infection and delayed healing.

Patients under the age of 7 have the highest risk of complications. One explanation for this is that young children are more prone to expander rupture due to external pressure on the expanded skin. Expansion in the extremities caries twice the risk of complication compared to other regions. The use of tissue expansion in congenital nevi has a 5-7\% complication rate. Tissue that has undergone serial expansion (two or more prior expansions) is at a higher risk for a major complication.

\section{Surgery of primary melanoma}

Pediatric melanoma is rare but increasing in incidence [63] limited options are possible for treatment. Early diagnosis and surgical management are the cornerstone of therapy and must adhere to the guidelines estabilished by the American Joint Committee on Cancer (AJCC) [64] Diagnosis of melanoma in children is more difficult than in adults, it relates to a number of variables, so many criteria used in adults are of limited value, for example the natural evolution of congenital and acquired nevi during childhood and adolescence [65] Historically, a wide excision with 5-cm margins with regional lymph node dissection was recommended for all melanomas. This indication, dating back 1907, was based on evaluations following a single necropsy, on a patient with advanced melanoma, assuming that in this way, all possible neoplastic foci would have been eliminated.

Furthermore, the indications suggested to extend the excision below the fascia, so as to also remove the superficial vascular and lymphatic structures.

This attitude has remained unchanged, until Breslow and Match described the treatment of melanoma with narrow margins [66] 
Once decided to remove a suspicious lesion, it is recommended to perform a 1- to 2-mm circumferential margin. There are no prospective data to provide an evidence-based approach in this setting.

It has long been suggested that malignant cells may be shed into the bloodstream during any given surgical procedure for cancer. While there is no evidence to suggest that an incisional biopsy does cause local spread of melanoma, it is generally not advocated. [67]

There may be times that the incision is to big that the tissue are not able to cover the skin defect, in this case is possible to use a skin graft, or in order to avoid a graft, the surgical defect may be closed using a rotational or advancement flap

The orientation of the incision should follow the relaxed skin tension lines (RSTLs, also known as lines of Langer), however, at the level of the limbs incisions parallel to the major axis of the limb are used, not to alter the paths of lymphatic drainage.

In the setting of dysplastic changes or once the diagnosis of melanoma is established, in children, surgical excision should be performed with the same excision margins recommended for adults by the National Comprehensive Cancer Network (NCCN) in 2007, and depends on the Breslow depth of the primary lesion, Clark's level of tumor invasion may provide additional prognostic value for thin melanomas [68].

The basic oncologic criteria of surgery are: the resection margins and the depth of the skin excision.

When there is an in situ melanoma, excision should include 0,5 centimeter of normal skin surronding the tumor and takes off the skin layers down to the fat; in removing an invasive melanoma that is $2 \mathrm{~mm}$ thick the margins are extended to $1 \mathrm{~cm}$ and the excision goes through all skin layers and down to the fascia; margins are $2 \mathrm{~cm}$ for lesions greater than $2 \mathrm{~mm}$ in thickness. [69]

The depth of excision can reach muscolaris fascia, whose removal has no oncological meaning, furthermore the preservation of the fascia allows a better aesthetic result.

An exception is the localization to the face, in these cases also with melanomas more than 4 $\mathrm{mm}$ thick margins of $1 \mathrm{~cm}$ are used.

In recent years, great importance was served to sentinel lymph node biopsy (SLNB) for the detection of lymph node metastases, in fact in adult melanoma therapy, it has become a mandatory procedure in the current AJCC staging system; however its use in the pediatric population has been limited.

Lymph node are the most common site of initial metastases [70], the lack of disease in the sentinel lymph node should indicate the lack of dissemination.

SLNB will select, with a minimally invasive technique, patients who should undergo regional lymph node dissection for clinically occult loco-regional metastases, so as to avoid completion lymph node dissection if the sentinel node is negative. 
SLNB was first described by Morton et al. in 1992 [71] The procedure is usually performed concurrent with re-excision of the primary lesion, and is advised for lesions thicker than $1 \mathrm{~mm}$ or for those between $0,76 \mathrm{~mm}$ and $1 \mathrm{~mm}$ with ulceration or reticular dermal invasion.

The procedure involves injection of the primary cutaneous lesion site with technectium-99m sulfur colloid followed by lymphoscintigraphy in the nuclear medicine suite. This is done on the morning of scheduled re-excision, and the patient is brought to the operating room in the afternoon. The lesion is injected with approximately $1 \mathrm{ml}$ of $1 \%$ isosulfan blue dye. The dye is allowed to travel through lymphatics for several minutes, and a hand held gamma counter is used to determine the area of maximal radiolabeled tracer intensity for lymph node sampling. An incision is made over the area identified to have the most active uptake of radiolabeled tracer as determined by the handled gamma probe and the preoperative lymphoscintigraphy. Upon examination of the draining lymph node basin, all nodes that are blue, palpable, or show significant activity with the gamma probe are excised and sent fresh to pathology [72].

The incision must be oriented so as to allow an eventual loco-regional lymphadenectomy, the lymph node is identified with the gamma camera and visually with the blue dye; the lymph node is removed after ligation of the afferent and efferent lymphatic vessels, after the removal it is necessary to evaluate "ex vivo" the radioactivity of the lymph node and the possible presence of other involved nodes.

A small lymphocele may result in postoperative period, usually with the possibility of spontaneous regression.

Elective regional lymph node dissection is subsequently performed if the result of the SLNB is positive for metastases [38].

\section{Treatment of metastatic disease}

\subsection{Congenital melanoma and transplacental metastases}

Congenital melanoma as a result of placental transmission from a mother with metastatic melanoma is extremely rare, with only a few cases described in literature [73, 74, 75, 76, 77]

To date, metastatic disease transmission from fetus to mother has never been reported. $[78,79]$

\subsection{Lymph node metastases}

\subsubsection{Staging}

After primary surgery and diagnosis of melanoma, staging of the disease is completed by pathologic detection of lymphatic involvement. Comprehensive staging guidelines for paediatric and adolescent melanoma have not been clearly established.

The American Joint Committee on Cancer (AJCC) provides a reproducible model on the natural history of melanoma and a detailed description of important prognostic variables. For 
localised disease, ulceration has been recognized as an important predictor of outcome and growing consideration is given to the significance of melanoma thickness. New importance has been recognized to the number of lymph nodes involved, the significance of in-transit or satellite metastases, the description of the sites of metastases and the prognostic value of serum lactic dehydrogenase [23].

Future trials including paediatric and adolescent melanoma patients should incorporate this new staging system to achieve a wider interpretation of results from institutions and patient populations. In addition, the routine use of sentinel node biopsy for the staging of paediatric and adolescent melanoma is mandatory, in order to determine the prognostic and therapeutic value of this procedure in young patients and to compare these results with those reported in the adult literature [80]

Although in adult patients the routine use of chest and abdomen computed tomography is not recommended in literature, in paediatric patients it has been found useful in about $25 \%$ of cases to identify clinically undetectable metastases from thick localised melanomas or patients with melanoma arising at an unknown primary site [81, 82]

The routine use of magnetic resonance imaging (MRI) to detect brain metastases is not advocated. For localised lesions under $1.5 \mathrm{~mm}$ thick, investigations include a complete blood count, serum chemistries including liver function tests, and a chest radiograph.

Positron emission tomography (PET) is a very useful tool in adults, but its use in paediatric patients has not been validated [23]

\subsubsection{Sentinel lymph nodes}

Early primary excision of melanoma is the mainstay of definitive treatment of the tumour. With the introduction of sentinel lymph node biopsy (SLNB) the treatment of patients with melanoma has been revolutioned.

The adoption of SLNB has led to selection of patients who do not need elective lymph node dissection (ELND) and in which the morbidity linked to this procedure can be avoided. The techniques of preoperative lymphoscintigraphy and sentinel lymph node (SLN) biopsy have become the standard of care for staging adult patients after detection of a primary melanoma. SLNB is particularly important in intermediate-thickness $(1.2-3.5 \mathrm{~mm})$ primary melanomas in order to indicate elective lymphadenectomy and has also a prognostic value [83]. SLNB is a very promising technique also in paediatric patients $[84,85,86]$. However, due to paucity of available data, the role of SLNB in paediatric patients is still debated, as concerns both its prognostic [87] and therapeutic implications.

SLN biopsy should be included in the surgical management of children. The indications for SLN biopsy in paediatric and adolescent patients are based on the adult literature and include the presence of lesions thicker than $1 \mathrm{~mm}$, the presence of ulceration or a Clark's level of invasion of IV or V in patients with lesion thickness of less than $1 \mathrm{~mm}$. The technique is the same as in adults. Excision with $2 \mathrm{~mm}$ margins of normal skin is performed. After diagnosis of melanoma, the patient undergoes SLNB for tumour thickness $\geq 1 \mathrm{~mm}$ followed by wide 
excision of the tumour site with $2 \mathrm{~cm}$ margins and primary closure or skin graft. SLNB is performed using preoperative lymphoscintigraphy, intraoperative blue dye injection around the site of excision and hand-held gamma probe for radio-localization $[38,88]$. One day before the operation, between 18.5 and $40 \mathrm{MBq}$ of Tc- $99 \mathrm{~m}$ microcolloid is injected intradermally around the scar. The drainage of the colloid is localized by detecting radiation, and the location of the SLN is marked on the skin. The position of the SLN is confirmed with a handheld gamma probe before starting the operation. At the author's center the procedure is performed under epidural anaesthesia and sedation or general anaesthesia. As reported by some authors, subcutaneous infusion anaesthesia (SIA) can be useful [89]. Patent blue is additionally injected intradermally around the scar as standard procedure. Sentinel lymph node biopsy is then accomplished with the help of repeated measurements with the handheld gamma probe. The $\mathrm{SLN}(\mathrm{s})$ is (are) removed, and the wound is closed.

A comparison between adults and patients younger than 21 years who underwent either lymph node dissection or SLNB showed a higher rate of lymph node metastasis in the paediatric age (44\%) as compared to the adult $(23.9 \%)$. However this finding had no statistical significance. In this series, paediatric patients either with Stage I or Stage II disease showed a $94.4 \%$ 10-year survival, while patients with Stage III melanoma had a $60.1 \%$ 10-year survival [90]

Recent data show that although the SLNB positivity rate is higher in paediatric and adolescent melanoma patients than in adults, non SLNB positivity and melanoma specific death rate are low [91]

\subsubsection{Regional lymph nodes}

In case of positive SLNB many surgeons would proceed to a completion lymph node dissection (CLND),

however survival advantage of this procedure is unclear, and is currently being investigated $[92,93,94]$

In a large series of paediatric melanoma cases 18 patients underwent SNLB, and 7 proceeded to undergo CLND because of findings of metastatic disease to the SLN; two of these had tumour-positive lymph nodes on pathologic analysis of the CLND specimen.

Similarly, the presence of metastases in regional lymph nodes after CLND has been diagnosed in 1 of 3 patients by some authors and in 1 of 4 patients by others [38, 92]

\subsubsection{Adjuvant therapy}

Consideration of systemic therapy after regional lymph nodes involvement by melanoma cells is under investigation. Treatment plans for children must be extrapolated from adult studies.

Interferon alfa-2b is currently used for adjuvant therapy in high-risk melanoma after surgery in adult patients and can also be used in paediatric melanoma patients with acceptable toxicity [95] 


\subsection{Distant metastases}

The incidence of metastatic melanoma has increased over the last three decades, and the death rate continues to climb faster than that of most other cancers. According to the American Cancer Society, there were approximately 68,000 new cases of melanoma in the United States in 2009, and 8,700 melanoma-related deaths. Melanoma is difficult to treat once it has spread beyond the skin to other parts of the body (metastasized). Very few treatment options exist for people with metastatic melanoma.

\subsubsection{Treatment of disseminated disease}

Most reports describing the treatment of paediatric melanoma are from single institutions in which diagnostic criteria, staging and pathological evaluation of the primary tumour have varied significantly. Dacarbazine, which is the most active agent in adult melanoma, showed encouraging activity in four children with melanoma treated between 1975 and 1984 [96] Other traditional chemotherapeutic regimens have shown some efficacy in metastatic melanoma [23]. The availability of investigational therapies, such as interleukin-2, interferon alfa-2b and vaccines, has been generally restricted to patients who are older than 18 years of age and no prospective trials in adolescents have been performed. Collaborative efforts, now under discussion between paediatric and adult cooperative groups, should help facilitate the enrollment of younger patients onto trials that use experimental therapies.

\subsubsection{Radiotherapy}

Radiotherapy is rarely indicated in the management of primary paediatric melanoma. However, it should be considered in patients with head and neck melanomas at high risk for parotid or cervical metastases and in those who develop brain metastases. Brain metastases have been reported to occur during the course of the disease in up to $18 \%$ of children with melanoma [23]. Ultimately, as in adults, there is no effective therapy for metastatic melanoma in children. Therefore, the main focus of the parent, the paediatrician, and the dermatologist should be risk reduction and early detection of melanoma. The former consists primarily of avoiding intense sunlight exposure, using protective clothing and broad-spectrum sunblock, and educating children. Early detection requires a high index of clinical suspicion, especially by the paediatrician, who sees children with much more regularity than a dermatologist, of any rapidly growing or otherwise atypical pigmented lesion. In addition, the physician should recognize the elevated risk of any child with a family history of melanoma, GCMN, or dysplastic nevi. Again, prevention and early clinical diagnosis are the only current effective cure for cutaneous melanoma [97].

\subsection{Prognosis}

The outcome for children and adolescents with melanoma also appears to be similar to that reported for adults and is dependent on the initial stage of the tumour. Patients with localised disease have an excellent outcome, whereas those with nodal and distant metastases have estimated 10-year survivals of only 60 and $25 \%$, respectively. Outcome is also stage-dependent 
and the thickness of the primary lesion correlates with the risk of nodal involvement and subsequent disease recurrence [23]

Melanomas arising on congenital nevi seem to have a better prognosis if they arise during early infancy than in childhood; moreover, metastatic melanoma associated with giant nevi have a worse prognosis than those associated with other skin lesions [32]

Melanoma has also reported to be more frequently metastatic in young children than in adolescents.This can be due to several causative factors but can also reflect a true biologic difference [98, 99]. There were significant differences in baseline characteristics of young children (age $<10$ years) compared with adolescents and young adults: the former were more likely to be non-white, to have metastases, to have nodular or other histology, head, face, or neck primaries, thicker lesions and history of cancer.

Multivariate analysis for melanoma survival in children showed significantly worse survival for males, patients with regional or unstaged disease, nodular histology, increasing thickness of the primary tumor, primary disease in the head, face, neck, eye, orbit, central nervous system, genitals, or overlapping sites, earlier year of diagnosis and previous cancer. Five-year melanoma-specific survival for pediatric cases (age $<20$ years) was 100\% for in situ disease, $96.1 \%$ for localized disease, $77.2 \%$ for regional disease and $57.3 \%$ for distant disease. Five-year overall survival was $88.9 \%$ for young children (age $<10$ years), $91.5 \%$ for adolescents (age 10 to 19 years) and $90.9 \%$ for young adults, but the latter data had not statistical significance [100]. Recent data confirm that paediatric melanoma patients in younger ages have an increased risk of lymph node metastasis and thicker tumors. This suggests that the younger paediatric patients may have a disease that differs biologically from that of the older ones [101].

\section{Author details}

Andrea Zangari ${ }^{1,2,3}$, Federico Zangari ${ }^{1,2,3}$, Mercedes Romano ${ }^{1,2,3}$, Elisabetta Cerigioni ${ }^{1,2,3}$, Maria Giovanna Grella ${ }^{1,2,3}$, Anna Chiara Contini ${ }^{1,2,3}$ and Martino Ascanio ${ }^{1,2,3}$

1 Pediatric Surgery Department, University Hospital of Ancona, Italy

2 San Camillo Hospital, Roma, Italy

3 Catholic University of the Sacred Heart, Roma, Italy

\section{References}

[1] Clemmensen, O. J, \& Kroon, S. The histology of "congenital features" in early acquired melanocytic nevi. J Am Acad Dermatol. Oct (1988). , 19(4), 742-6. 
[2] Krengel, S, Hauschild, A, \& Schafer, T. Melanoma risk in congenital melanocytic naevi: a systematic review. Br J Dermatol. Jul (2006). , 155(1), 1-8.

[3] Ansarin, H, Soltani-arabshahi, R, Mehregan, D, Shayanfar, N, \& Soltanzadeh, P. Giant congenital melanocytic nevus with neurofibroma-like changes and spina bifida occulta. Int J Dermatol. Nov (2006). , 45(11), 1347-50.

[4] Cruz, M. A, Cho, E. S, Schwartz, R. A, \& Janniger, C. K. Congenital neurocutaneous melanosis. Cutis. Oct (1997). , 60(4), 178-81.

[5] Silfen, R, Skoll, P. J, \& Hudson, D. A. Congenital giant hairy nevi and neurofibromatosis: the significance of their common origin. Plast Reconstr Surg. Oct (2002). , 110(5), 1364-5.

[6] Cramer, S. F. The melanocytic differentiation pathway in congenital melanocytic nevi: theoretical considerations. Pediatr Pathol. (1988). , 8(3), 253-65.

[7] Kinsler, V. A, Abu-amero, S, Budd, P, Jackson, I. J, Ring, S. M, Northstone, K, et al. Germline Melanocortin-Receptor Genotype Is Associated with Severity of Cutaneous Phenotype in Congenital Melanocytic Nevi: A Role for MC1R in Human Fetal Development. J Invest Dermatol. May 10 (2012). , 1.

[8] Kadonaga, J. N, \& Frieden, I. J. Neurocutaneous melanosis: definition and review of the literature. J Am Acad Dermatol. May (1991). Pt 1):747-55.

[9] Bousema, M. T, et al. Non-von Recklingausen's resembling a giant pigmented nevus. J Am Acad Dermatol 20:358, 1989

[10] Everett, M. A. Histopathology of congenital pigmented nevi. Am J Dermatopathol. Feb (1989). , 11(1), 11-2.

[11] Fitzpatrick, s, et al. Dermatology in General Medicine. Vol. I: 1028, (1999).

[12] Fitzpatrick, s, et al. Dermatology in General Medicine. Vol. I: 1030, (1999).

[13] Wu, P. A, Mancini, A. J, Marghoob, A. A, \& Frieden, I. J. Simultaneous occurrence of infantile hemangioma and congenital melanocytic nevus: Coincidence or real association?. J Am Acad Dermatol. Feb (2008). Suppl):S, 16-22.

[14] Changchien, L, Dusza, S. W, Agero, A. L, et al. Age- and site-specific variation in the dermoscopic patterns of congenital melanocytic nevi: an aid to accurate classification and assessment of melanocytic nevi. Arch Dermatol. Aug (2007). , 143(8), 1007-14.

[15] Hale, E. K, Stein, J, Ben-porat, L, et al. Association of melanoma and neurocutaneous melanocytosis with large congenital melanocytic naevi--results from the NYULCMN registry. Br J Dermatol. Mar (2005). , 152(3), 512-7.

[16] Fitzpatrick, s, et al. Dermatology in General Medicine. Vol. I: 1034, (1999).

[17] Fitzpatrick, s, et al. Dermatology in General Medicine. Vol. I: 1018-1025, (1999). 
[18] Fitzpatrick, s, et al. Dermatology in General Medicine. Vol. I: 1037-1041, (1999).

[19] Rigel, D. S, et al. The incidence of malignant melanoma in the United States:Issues As we approach the $21^{\text {st }}$ century. J Am Acad Dermatol 34:839, (1996).

[20] Cosary, C. L, et al. SEER Cancer Statistics Review, 1973-1992: National Cancer Institute, NRH Pub. Bethesda, Maryland, (1995). (96-2789), 96-2789.

[21] Ries, L, Eisner, M, Kosary, C, et al. SEER Cancer Statistics Review Bethesda, MD, National Cancer Institute, (2004). http://seer.cancer.gov/csr/1975_2001/, 1975-2000.

[22] Pappo, A. S. Ries LAG, Herzog C, et al: Malignant melanoma in the first three decades of life: A report from the U.S. Surveillance, Epidemiology and End Results (SEER) program. 23:721, (2004). abstr 7557)

[23] Pappo AS: Melanoma in children and adolescentsEur J Cancer 39:2651-2661, (2003).

[24] Kraemer, K. H, Lee, M. M, Andrews, A. D, et al. The role of sunlight and DNA repair in melanoma and nonmelanoma skin cancer: The xeroderma pigmentosum paradigm. Arch Dermatol 130:1018-1021, (1994).

[25] Tucker, M. A. Goldstein AM: Melanoma etiology: Where are we? Oncogene , 22, 3042-3052.

[26] Whiteman, D. C, Valery, P, Mcwhirter, W, et al. Risk factors for childhood melanoma in Queensland, Australia. Int J Cancer 70:26-31, (1997).

[27] Ahmed I: Malignant Melanoma: Prognostic IndicatorsMayo Clin Proc 72:356-361, (1997).

[28] Kaskel, P, Sander, S, Kron, M, et al. Outdoor activities in childhood: A protective factor for cutaneous melanoma? Results of a case-control study in 271 matched pairs. $\mathrm{Br}$ J Dermatol 145:602-609, (2001).

[29] Saenz, N. C, Saenz-badillos, J, Busam, K, et al. Childhood melanoma survival. Cancer 85:750-754, (1999).

[30] Milton, G. W, Shaw, H. M, Thompson, J. F, et al. Cutaneous melanoma in childhood: Incidence and prognosis. Australas J Dermatol 38:SS48, (1997). suppl 1), 44.

[31] Fears, T. R, Bird, C. C, Guerry, D I. V, et al. Average midrange ultraviolet radiation flux and time outdoors predict melanoma risk. Cancer Res , 62, 3992-3996.

[32] Tannous, Z. S. Mihm Jr MC, Sober AJ, et al Congenital melanocitic nevi: clinical and istopathologic features, risk of melanoma, and clinical management. J Am Acad Dermatol (2005). , 52(2), 197-203.

[33] Michel, J. L, Chalencon, F, Gentil-perret, A, et al. Congenital pigmented nevus: prognosis and therapeutic possibilities. Arch Pediatr (1999). , 6(2), 211-7. 
[34] Ruiz-maldonado, R, Tamayo, L, Laterza, A. M, et al. Giant pigmented naevi: clinical histopatologic and therapeutic considerations. J Pediatr (1992).

[35] Zaal, L. H, \& Mooi, W. J. Sillevis Smith JH, et al. Classification of congenital melanocytic naevi and malignant transformation: a review of the literature. Br J Plast Surg (2004). , 57(8), 707-19.

[36] Yesudian, P. D. Parslew RAG. A guide to the management of pigmented skin naevi in children. Curr Paediatr (2003). , 13, 407-12.

[37] Saenz, N. C, Saenz-badillos, J, Busam, K, et al. Childhood melanoma survival. Cancer (1999). , 85, 750-4.

[38] Zangari, A, Bernardini, M. L, Tallarico, R, Ilari, M, Giangiacomi, M, \& Offidani, A. M. Martino A: Indications for excision of nevi and melanoma diagnosed in a pediatric surgical unit. J Pediatr Surg. (2007). Aug;, 42(8), 1412-6.

[39] Gelbard, S. N, Tripp, J. M, Marghoob, A. A, et al. Management of Spitz nevi: a survey of dermatologists in the United States. J Am Acad Dermatol (2002). , 47(2), 224-30.

[40] Dyon GCTMSnels MD, Elysèe TM, et al. Risk of cutaneous malignant melanoma in patients with nonfamilial atypical nevi from a pigmented lesions clinic. Leiden, The Netherlands. J Am Acad Dermatol (1999). , 40, 686-93.

[41] Zaal, L. H, Mooi, W. J, Klip, H, et al. Risk of malignant transformation of congenital melanocytic nevi: a retrospective nationwide study from The Netherlands. Plast Reconstr Surg. Dec (2005). , 116(7), 1902-9.

[42] Rhodes, A. R, \& Melski, J. W. Small congenital nevocellular nevi and the risk of cutaneous melanoma. J Pediatr. Feb (1982). , 100(2), 219-24.

[43] Sahin, S, Levin, L, Kopf, A. W, et al. Risk of melanoma in medium-sized congenital melanocytic nevi: a follow-up study. J Am Acad Dermatol. Sep (1998). , 39(3), 428-33.

[44] Kearney-cooke, A. (2002). Familial influences on body image development. In T. F. Cash \& T. Pruzinsky (Eds.), Body image: A handbook of theory, and clinical practice (New York: Guilford., 99-107.

[45] Bradbury, E. (1997). Understanding the problems. In R. Lansdown, N. Rumsey, E. Bradbury, A. Carr, \& J. Partridge (Eds.), Visibly different: Coping with disfigurement (Oxford: Butterworth-Heinemann., 180-193.

[46] Turner, S, Thomas, P, Dowell, T, Rumsey, N, \& Sandy, J. (1997). Psychological outcomes amongst cleft patients and their families. British Journal of Plastic Surgery, , $50,1-9$.

[47] Gilbert, S, \& Thompson, J. (2002). Body shame in childhood \& adolescence. In P. Gilbert \& J. Miles (Eds.), Body Shame (Hove: Brunner-Routledge., 55-74. 
[48] Harter, S. (1999). The construction of the self: A developmental perspective. New York: Guilford.

[49] Hearst, D, \& Middleton, J. (1997). Psychological intervention and models of current working practice. In R. Lansdown, N. Rumsey, E. Bradbury, A. Carr, \& J. Partridge (Eds.), Visibly different: Coping with disfigurement (Oxford: Butterworth-Heinemann., 158-171.

[50] Bauer, B. S, \& Corcoran, J. Treatment of large and giant nevi. Clin Plast Surg. Jan (2005). vii., 32(1), 11-8.

[51] Margulis, A, Bauer, B. S, \& Fine, N. A. Large and giant congenital pigmented nevi of the upper extremity: an algorithm to surgical management. Ann Plast Surg. Feb (2004). , 52(2), 158-67.

[52] Arneja, J. S, \& Gosain, A. K. Giant congenital melanocytic nevi. Plast Reconstr Surg. Aug (2007). e-40e.

[53] Bauer, B. S, \& Corcoran, J. Treatment of large and giant nevi. Clin Plast Surg. Jan (2005). vii., 32(1), 11-8.

[54] Pearson, G. D, Goodman, M, \& Sadove, A. M. Congenital nevus: the Indiana University's approach to treatment. J Craniofac Surg. Sep (2005). , 16(5), 915-20.

[55] Rumsey, N, \& Harcourt, D. Body image and disfigurement: issues and interventions. Body Image (2004). , 1(2004), 83-97.

[56] Vinod K JainMahendra K Singhi, and Rajiv Goyal: Serial Excision of Congenital Melanocytic Nevi. J Cutan Aesthet Surg. (2008). January; , 1(1), 17-18.

[57] Gosain, A. K, Santoro, T. D, \& Larson, D. L. Gingrass RP Giant congenital nevi: a 20year experience and an algorithm for their management. Plast Reconstr Surg. (2001). Sep $1 ;$, 108(3), 622-36.

[58] Pasyk, K. A, Argenta, L. C, \& Hassett, C. Quantitative analysis of the thickness of human skin and subcutaneous tissue following controlled expansion with a silicone implant. Plast Reconstr Surg. Apr (1988). , 81(4), 516-23.

[59] Timothy, M. Johnson, Md Lori Lowe, Md Marc D. Brown, Md Michael J. Sullivan, Md Bruce R. Nelson, Md: Histology and Physiology of Tissue Expansion. J Dermatol Surg Oncol (1993). , 19, 1074-1078.

[60] Chao, J. J, Longaker, M. T, \& Zide, B. M. Expanding horizons in head and neck expansion. Copyright (1998). by W.B. Saunders Company

[61] Farhad HafeziBijan Naghibzadeh, Mohammad Pegahmehr, Amirhossein Nouhi: Use of overinflated tissue expanders in the surgical repair of head and neck scars Journal of Plastic, Reconstructive \& Aesthetic Surgery ((2008). xx, 1e8

[62] BauerBruce S. M.D.; Few, Julius W. M.D.; Chavez, C. D. M.D., and; Galiano, R. D. B.A.: The Role of Tissue Expansion in the Management of Large Congenital Pigment- 
ed Nevi of the Forehead in the Pediatric Patient. Plastic \& Reconstructive Surgery: March (2001). Articles(3-pp), 668-675.

[63] Lange, J. R, Palis, B. E, Chang, D. C, Soong, S-J, \& Balch, C. M. melanoma in children and teenagers: an analysis of patients from the national cancer data base. J Clin Oncol (2007). , 25(11), 2007-1363.

[64] Balch, C. M, Buzaid, A. C, Soong, S-J, Atkins, M. B, \& Cascinelli, N. et alii Final Version of the American Joint Committee on Cancer Staging System for Cutaneous Melanoma. J Clin Oncol, 19, (16) (2001).

[65] Huynh, P. M, Grant-kels, J. M, \& Grin, C. M. Childhood melanoma: update and treatment. Int J Dermatol (2005).

[66] Eedy, D. J. Surgical treatment of melanoma. Brit J Dermatol 149, (2003).

[67] Swanson, N. A. Lee Kl, Gorman A, Lee HN. Biopsy techniques: diagnosis of melanoma. Dermatol Clin 20, (2002).

[68] NCCNNCCN clinical practice guidelines in oncology: melanoma (Washington, DC: $\operatorname{NCCN}(2008) ., 2$

[69] Swetter, S. M. Malignant melanoma. eMedicine.com, Inc.(2004).

[70] Faries, M. B, \& Morton, D. L. Surgery and sentinel lymph node biopsy. Semin Oncol (2007). December; , 34(6), 498-508.

[71] Morton, D. L, Wen, D. R, \& Wong, J. H. Technical details of intraoperative lymphatic mapping for early stage melanoma. Arch Surg (1992). , 127, 392-399.

[72] Downard, C. D, Rapkin, L. B, \& Gow, K. W. Melanoma in children and adolescents. Surgical Oncol ((2007).

[73] Wiggins, C. L, Berwick, M, et al. Malignant Melanoma in Pregnancy Newton Bishop Obstet Gynecol Clin N Am(2005). , 32(2005), 559-568.

[74] Richardson, S. K, \& Tannous, Z. S. Mihm Jr MC. Congenital and infantile melanoma: review of theliterature and report of an uncommon variant, pigment-synthesizing melanoma. J Am AcadDermatol (2002). , 47, 77-90.

[75] Alexander, A, Samlowski, W. E, Grossman, D, et al. Metastatic melanoma in pregnancy: risk oftransplacental metastases in the infant. J Clin Oncol (2003). Baergen RN, Johnson D, Moore T, et al. Maternal melanoma metastatic to the placenta: a case report and review of the literature. Arch Pathol Lab Med 1997;121:508-11;,21, 2179-86.

[76] Dildy III GAMoise Jr KJ, Carpenter Jr RJ, et al. Maternal malignancy metastatic to the products of conception: a review. Obstet Gynecol Surv (1989). Dargeon HW, Eversole J, Del Duca V. Malignant melanoma in an infant. Cancer 1950;3:299-306;, 44, 535-40.

[77] Brodsky, I, Baren, M, \& Kahn, S. B. Lewis G Jr, Tellem M. Metastatic malignant melanoma from mother to fetus. Cancer (1965). , 18, 1048-54. 
[78] Richardson, S. K, \& Tannous, Z. S. Mihm Jr MC. Congenital and infantile melanoma: review of the literature and report of an uncommon variant, pigment-synthesizing melanoma. J Am Acad Dermatol (2002). , 47, 77-90.

[79] Brenn, T. Phillip H McKee P: Melanoma in children and adolescents Diagnostic Histopathology, January (2008). , 14(1), 18-27.

[80] Kogut, K. A, Fleming, M, Pappo, A. S, et al. Sentinel lymph node biopsy for melanoma in young children. J Pediatr Surg (2000). , 35, 965-6.

[81] Buzaid, A. C, Sandler, A. B, Mani, S, et al. Role of computed tomography in the staging of primary melanoma. J Clin Oncol (1993). , 1993(11), 638-643.

[82] Kaste, S. C, \& Pappo, A. S. Jenkins III JJ, Pratt CB. Malignant melanoma in children: imaging spectrum. Pediatr Radiol (1996). , 1996(26), 800-805.

[83] Morton, D. L, Thompson, J. F, Cochran, A. J, et al. MSLT Group. Sentinel node biopsy or nodal observation in melanoma. N Engl J Med (2006). , 355, 1307-17.

[84] Roaten, J. B, Partrick, D. A, Pearlman, N, et al. Sentinel lymph node biopsy for melanoma and other melanocytic tumors in adolescents. J Pediatr Surg (2005). , 40, 232-5.

[85] Toro, J, Ranieri, J. M, Havlik, R. J, et al. Sentinel lymph node biopsy in children and adolescents with malignant melanoma. J Pediatr Surg (2003). , 38, 1063-75.

[86] Pacella, S. J, Lowe, L, Bradford, C, et al. The utility of sentinel lymph node biopsy in head and neck melanoma in the pediatric population. Plast Reconstr Surg (2003). , $112,1257-65$.

[87] Roka, F, Kittler, H, Cauzig, P, et al. Sentinel node status in melanoma patients is not predictive for overall survival upon multivariate analysis. Br J Cancer (2005). , 92, 662-7.

[88] Butter AndreanaHuib Tom, Chapdelaine Joyaube, Beaunoyer Mona, Flageole Helene, Bouchard Sarah: Melanoma in children and the use of sentinel lymph node biopsy. Journal of Pediatric Surgery ((2005).

[89] Topar GerdaZelger Bernhard: Assessment of value of the sentinel lymph node biopsy in melanoma in children and adolescents and applicability of subcutaneous infusion anestesia Journal of Pediatric Surgery ((2007).

[90] Livestro, D. P, Kaine, E. M, Michaelson, J. S, et al. Melanoma in the young: differences and similarities with adult melanoma: a case-matched controlled analysis. Cancer (2007). , 110, 614-24.

[91] Robert Howman-GilesMD et Al. Sentinel Lymph Node Biopsy in Pediatric and Adolescent Cutaneous Melanoma Patients. Ann Surg Oncol ((2010). 
[92] Gow, K. W, Rapkin, L. B, Olson, T. A, Durham, M. M, \& Wyly, B. Shehata BM: Sentinel lymph node biopsy in the pediatric population. Journal of Pediatric Surgery ((2008).

[93] Sabel, M. S, \& Arora, A. The role of the surgeon in the management of melanoma. Minerva Chir (2006). , 61, 54-141.

[94] Mcmasters, K. M, Reintgen, D. S, Ross, M. I, et al. Sentinel lymph node biopsy for melanoma: controversy despite widespread agreement. Journal of Clinical Oncology (2001). , 19, 2851-5.

[95] Shah, N. C, Gerstle, J. T, Stuart, M, et al. Use of sentinel lymph node biopsy and highdose interferon in pediatric patients with high-risk melanoma: the Hospital for Sick Children experience. J Pediatr Hematol Oncol (2006). , 28, 496-500.

[96] Boddie AW JrCangir A. Adjuvant and neoadjuvant chemotherapy with dacarbazine in high-risk childhood melanoma. Cancer. (1987). Oct 15;,60(8), 1720-3.

[97] Fishman, C, \& Mihm, M. C. Sober AJ: Diagnosis and Management of Nevi And Cutaneous Melanoma in Infants And Children. Clinics in Dermatology Y (2002). , 20, 44-50.

[98] Bressac-de-paillerets, B, Avril, M. F, Chompret, A, \& Demenais, F. Genetic and environmental factors in cutaneous malignant melanoma. Biochimie. (2002). , 84, 67-74.

[99] Brenn, T. McKee PH: Melanoma in children and adolescents Diagnostic Histopathology, January (2008). , 14(1), 18-27.

[100] Strouse, J. J, Fears, T. R, \& Tucker, M. A. Wayne AS: Pediatric Melanoma: Risk Factor and Survival Analysis of the Surveillance, Epidemiology and End Results Database Journal of Clinical Oncology, July 20), (2005). , 23(21), 4735-4741.

[101] Moore-olufemi, S, Herzog, C, \& Warneke, C. Gershenwald Jerry E, Mansfield P, Ross M, Prieto V, Lally KP and Hayes-Jordan A: Outcomes in Pediatric Melanoma. Comparing Prepubertal to Adolescent Pediatric Patients. Annals of Surgery (2011). , 253(6), 1211-15. 
